\title{
First-principles energetics of water clusters and ice: A many-body analysis
}

\author{
M. J. Gillan, ${ }^{1,2,3, a)}$ D. Alfè,,$^{1,2,3,4}$ A. P. Bartók, ${ }^{5}$ and G. Csányi ${ }^{5}$ \\ ${ }^{1}$ London Centre for Nanotechnology, UCL, London WC1H OAH, United Kingdom \\ ${ }^{2}$ Thomas Young Centre, UCL, London WC1H OAH, United Kingdom \\ ${ }^{3}$ Department of Physics and Astronomy, UCL, London WC1E 6BT, United Kingdom \\ ${ }^{4}$ Department of Earth Sciences, UCL, London WC1E 6BT, United Kingdom \\ ${ }^{5}$ Department of Engineering, University of Cambridge, Cambridge, United Kingdom
}

(Received 2 October 2013; accepted 6 December 2013; published online 30 December 2013)

\begin{abstract}
Standard forms of density-functional theory (DFT) have good predictive power for many materials, but are not yet fully satisfactory for cluster, solid, and liquid forms of water. Recent work has stressed the importance of DFT errors in describing dispersion, but we note that errors in other parts of the energy may also contribute. We obtain information about the nature of DFT errors by using a many-body separation of the total energy into its 1-body, 2-body, and beyond-2-body components to analyze the deficiencies of the popular PBE and BLYP approximations for the energetics of water clusters and ice structures. The errors of these approximations are computed by using accurate benchmark energies from the coupled-cluster technique of molecular quantum chemistry and from quantum Monte Carlo calculations. The systems studied are isomers of the water hexamer cluster, the crystal structures Ih, II, XV, and VIII of ice, and two clusters extracted from ice VIII. For the binding energies of these systems, we use the machine-learning technique of Gaussian Approximation Potentials to correct successively for 1-body and 2-body errors of the DFT approximations. We find that even after correction for these errors, substantial beyond-2-body errors remain. The characteristics of the 2-body and beyond-2-body errors of PBE are completely different from those of BLYP, but the errors of both approximations disfavor the close approach of non-hydrogen-bonded monomers. We note the possible relevance of our findings to the understanding of liquid water. (C) 2013 AIP Publishing LLC. [http://dx.doi.org/10.1063/1.4852182]
\end{abstract}

\section{INTRODUCTION}

The pioneering work of Parrinello and co-workers ${ }^{1}$ in the 1990s initiated a major effort to understand the properties of water systems from first principles using density-functional theory (DFT). This effort is important not just for the pure liquid and solid, but for general aqueous systems, including solutions $^{2}$ and water on surfaces. ${ }^{3}$ However, standard DFT methods often give less than satisfactory predictions for water in its liquid, ${ }^{4-19}$ crystalline ${ }^{20-26}$ and cluster forms, ${ }^{27-30}$ for reasons that are still not generally agreed. ${ }^{26}$ Strenuous efforts have been devoted to the development of improved exchange-correlation functionals and empirical correction potentials, with a strong recent emphasis on the description of dispersion (non-local electron correlation). ${ }^{13-19,22-26,29-34}$ However, these efforts have met with only partial success, ${ }^{26}$ and it is not established that dispersion is the only important source of DFT errors for water systems. Here we show how a combination of correlated quantum chemistry, machine learning, and quantum Monte Carlo can be used to analyze DFT errors for water in a variety of aggregation states, focusing particularly on clusters and ice structures. With a many-body decomposition of the total energy, we find that the two commonly used density functionals $\mathrm{PBE}^{35}$ and $\mathrm{BLYP}^{36}$ suffer not only from 1- and 2-body errors, but also from substantial

\footnotetext{
a) Author to whom correspondence should be addressed. Electronic mail: m.gillan@ucl.ac.uk
}

beyond-2-body errors, which may not be associated with non-local correlation.

Many recent papers on the problems of DFT for water systems have been concerned with the thermodynamics, structure, and dynamics of the liquid at near-ambient conditions. ${ }^{4-14,16,18,19,33}$ Some of these papers assume that the poor description of dispersion is the main source of errors in describing the liquid. Liquid water is obviously important, but we have chosen to concentrate here on clusters and ice structures because they are somewhat easier to study and we consider them to be equally important. In addition, we deliberately avoid making any assumptions about what is wrong with standard DFT approximations. The errors of standard DFT methods for assemblies of water molecules show themselves in many ways, including: inaccurate predictions of monomer deformation energies ${ }^{37}$ and the energies for some dimer geometries; ${ }^{27,28,38}$ wrong stability ordering of isomers of some clusters, notably the hexamer, ${ }^{29,39}$ incorrect relative energies of different ice structures; ${ }^{23-26}$ errors of up to $30 \%$ in the predicted density of the liquid; ${ }^{14,15,19,40}$ and substantial errors in the structure and diffusivity of the liquid. ${ }^{4,14-16,19}$ An understanding of the relationships between these different errors is clearly needed, and the present work is intended to contribute to this understanding.

The many-body expansion that we use has long served $^{41,42}$ as a standard tool for analyzing the energetics of water clusters. It expresses the total energy $E_{\mathrm{tot}}(1,2, \ldots N)$ of 
a system of $N$ monomers as:

$$
\begin{aligned}
E_{\mathrm{tot}}(1,2, \ldots N)= & \sum_{i} E^{(1)}(i)+\sum_{i<j} E^{(2)}(i, j) \\
& +E^{(>2)}(1,2, \ldots N) .
\end{aligned}
$$

Here, $i$ is the collection of variables describing monomer $i$ (position, orientation, internal distortion from equilibrium geometry), $E^{(1)}(i)$ the 1-body (1B) energy of monomer $i$, $E^{(2)}(i, j)$ the 2-body (2B) interaction energy of monomers $i$ and $j$, and the beyond-2-body (B2B) energy $E^{(>2)}$ is everything not included in $1 \mathrm{~B}$ and $2 \mathrm{~B}$ energy. Electrostatic interactions between the electron distributions on the monomers, exchangerepulsion between these distributions, and non-local correlations of their fluctuations account for a large part of the $2 \mathrm{~B}$ interactions, with contributions also from polarizability and charge transfer (partial covalency). ${ }^{43}$ We know that B2B interactions are crucially important, because the dipole moment of the water molecule increases by at least $40 \%^{44}$ and perhaps as much as $65 \%$ or more ${ }^{18,45-48}$ when it passes from the gas phase to the ice and liquid phases. The importance of B2B energies is also apparent from the well-known cooperativity of hydrogen bonding, i.e., the non-additivity of hydrogen-bond energies, which has been extensively investigated for water and other systems for over 40 years. ${ }^{41,49-52}$ It is often assumed that polarizability gives the only important contribution to $E^{(>2)},{ }^{53-55}$ but it is likely that the electronic redistribution associated with the change of dipole moment affects exchange-repulsion, non-local correlation, and charge transfer. ${ }^{42}$ It is therefore important to understand the distribution of DFT errors between the terms of the many-body expansion.

Our plan in the present work is to analyze DFT errors for water clusters and ice structures by separating them into their 1B, 2B, and B2B parts. To do this, we use the machinelearning technique known as GAP (Gaussian Approximation Potentials). ${ }^{56}$ This Bayesian inference technique was originally designed to use data-bases of DFT energies and forces to train rapidly computable algorithms to perform simulations with essentially the same accuracy as the original data-bases. However, we have recently shown ${ }^{57}$ how the same ideas can be used to train algorithms for predicting the differences between DFT energies and forces and those computed using the accurate correlated quantum chemistry techniques MP2 and $\operatorname{CCSD}(\mathrm{T})$ (second-order Møller-Plesset and coupled-cluster singles, doubles and perturbative triples). ${ }^{58}$ The GAP methods allow us to create accurate representations of the 1B and 2B errors of any chosen DFT approximation, and hence to correct almost exactly for these errors. Provided we have accurate total energies for the systems we wish to study, we can then separate the DFT errors into their 1B, 2B, and B2B parts, thus gaining additional insight into the nature of the errors.

We have chosen to focus on the PBE and BLYP functionals for several reasons. First, these functionals have been among the most commonly used in DFT simulations of liquid water (see, e.g., Refs. 7, 8, 10, 11, 14, 15, and 17), as well as in studies of ice energetics. ${ }^{22,23,26}$ Second, the errors of the two functionals are known to be of rather different kinds. For example, it is well known that BLYP gives substantial under-binding for the water dimer, while PBE gives a very good binding energy, at least for the dimer in its global-minimum geometry. ${ }^{28,59}$ Our third reason for examining PBE and BLYP is connected with the description of exchange-repulsion. It has long been recognized that in generalized-gradient approximations (GGA) the dependence of the enhancement factor $F$ on reduced gradient $x$ in the exchange part of the functional strongly affects the description of the exchange interaction due to wave-function overlap between closed-shell molecules. ${ }^{60-62}$ (Recall that $x$ is defined as $|\nabla \rho| / \rho^{4 / 3}$, where $\rho$ is electron density, and enhancement factor $F(x)$ determines the dependence of the functional on the gradient.) The enhancement factors of BLYP and PBE differ greatly for large $x$ values, ${ }^{61}$ and we can regard the two functionals as representatives of high- and low- $F$ varieties of GGA functional. (There is a connection here with our second reason, since the high exchange-enhancement of BLYP appears to be responsible for its 2-body under-binding. ${ }^{61}$ )

In Sec. II, we give technical details of our $\operatorname{CCSD}(\mathrm{T})$, DFT, and QMC calculations on clusters and the DFT calculations on ice structures. We also provide a summary of the GAP techniques, and we show how they can be used to correct the BLYP and PBE approximations so that they give extremely accurate predictions for the energies of the water dimer in any configuration of interest. In Sec. III, we then use the isomers of the water hexamer to verify that the use of GAP corrections to analyze the errors of BLYP and PBE into their 1B, 2B, and B2B components reproduces the results known from earlier work. Following this, we use the same techniques to analyze the errors of these two DFT approximations for four important ice structures. We shall show that further insight into the errors for ice structures can be gained by studying clusters taken from the ice VIII structure. In Sec. IV, we discuss connections between our analysis and other recent work, and we also suggest the possible implications for the understanding of liquid water.

\section{TECHNIQUES}

\section{A. Coupled-cluster and DFT methods}

The absolute accuracy of basis-set converged CCSD(T) calculations ${ }^{58}$ on aggregates of water molecules is generally believed to be $\sim 0.1 \mathrm{~m} E_{\mathrm{h}}(\sim 3 \mathrm{meV})$ per monomer, ${ }^{63,64}$ and we use energies calculated with this technique as benchmarks for characterizing the errors of DFT approximations, which are typically an order of magnitude greater. ${ }^{27,28}$ Our benchmark $\operatorname{CCSD}(\mathrm{T})$ calculations on the dimer and hexamer clusters, as well as the DFT calculations on the dimers, hexamers, and larger clusters, were performed using the MOLPRO code. ${ }^{65,66}$

The coupled-cluster calculations on the dimers reported below employ the approach sometimes called "focal-point" analysis, ${ }^{64,67}$ in which the energies are first calculated with MP2 with large basis sets, and the energy difference $\triangle \operatorname{CCSD}(\mathrm{T})$ between $\operatorname{CCSD}(\mathrm{T})$ and MP2 is then computed using somewhat smaller basis sets. In both the MP2 and the $\operatorname{CCSD}(\mathrm{T})$ calculations, we use explicitly correlated (F12) methods, ${ }^{68-70}$ which greatly accelerate the convergence to the complete-basis-set (CBS) limit in correlated calculations and 
avoid the need for basis-set extrapolation. ${ }^{71} \mathrm{We}$ employed the standard Dunning ${ }^{72,73}$ correlation-consistent augmented basis sets aug-cc-pVQZ for the MP2 calculations, and augcc-pVTZ for the difference CCSDT - MP2. (For brevity, we refer to these basis sets as AVQZ and AVTZ.) The counterpoise technique ${ }^{74}$ was used to compute the 2-body energy. Our tests done with larger basis sets indicate that our benchmark 2-body energies for the dimer are within $\sim 40 \mu E_{\mathrm{h}}$ of the CBS limit of CCSD(T). (Numerical examples of the tests on which this statement is based are provided in the supplementary material. ${ }^{75}$ ) The PBE and BLYP 2-body energies of the dimers were computed using AVQZ basis sets with counterpoise. Tests on a subset of the configurations using AV5Z basis sets indicate that the residual basis-set error in the 2-body energy with AVQZ is less than $40 \mu E_{\mathrm{h}}$.

For the $\operatorname{CCSD}(\mathrm{T})$ benchmarks on the prism, cage, book, and ring isomers of the hexamer reported in Sec. III, we use a method analogous to the focal-point method. The total energies of the four isomers were first computed with MP2 using AVQZ basis sets and F12. We then added the 1-, 2-, and 3-body differences CCSD(T) - MP2. For the 1body correction, we actually used the exceedingly accurate Partridge-Schwenke 1-body energies ${ }^{90}$ rather than CCSD(T) values, though the differences between the two are negligible for present purposes. For the 2- and 3-body corrections, we used AVTZ basis sets with F12 and counterpoise. The PBE and BLYP energies of the four isomers were obtained from direct calculations on the entire hexamers using AV5Z basis sets. All the DFT calculations on the nonamer and decamer geometries were performed using AVQZ basis sets.

Our calculations on the XI, II, XV, and VIII crystal structures of ice using the PBE and BLYP functionals (Sec. III) were performed with the CASTEP package. ${ }^{76}$ The calculations employed on-the-fly generated ultra-soft pseudopotentials, $1200 \mathrm{eV}$ plane-wave cut-off and a $3 \times 3 \times 3$ Monkhorst-Pack $k$-point mesh in the primitive unit cell, both chosen to achieve $1 \mathrm{meV}$ convergence of total energies. The geometry and lattice parameters were relaxed until atomic displacements were smaller than $0.0001 \AA$ and the pressure was less than $0.1 \mathrm{GPa}$.

\section{B. Quantum Monte Carlo}

We have used the diffusion Monte Carlo (DMC) technique to obtain benchmark energies of the water clusters larger than the hexamer. The advantage of DMC for larger clusters is that its computational scaling with number of molecules is much milder than that of coupled-cluster methods, while for non-covalent interactions in water and other molecular systems, a considerable body of evidence $^{23,28,29,77-79}$ indicates that its accuracy approaches that of $\operatorname{CCSD}(\mathrm{T})$. Full information about DMC can be found in reviews. ${ }^{80-82}$

Our DMC calculations were performed with the CASINO code $^{83}$ within the usual fixed-node approximation ${ }^{84}$ and using B-spline basis sets. ${ }^{85}$ We used Dirac-Fock pseudopotentials, ${ }^{86}$ the oxygen having a frozen core with a radius of $0.4 \AA$ and the hydrogen pseudopotential having a core radius of 0.26 A. Pseudopotential non-locality was treated with the usual locality approximation. ${ }^{87}$ The trial wavefunctions were of Slater-Jastrow type, with a single Slater determinant. The single-electron orbitals were obtained from DFT-LDA planewave calculations using the PWSCF package, ${ }^{88}$ the planewave cut-off being $300 \mathrm{Ry}$, and the orbitals being re-expanded in B-splines. ${ }^{85}$ The time-step used was 0.002 a.u., which is the same as that used in Ref. 23. To check that this is short enough, we did tests on the nonamer geometries (see below) using times steps as small as 0.0005 a.u., and we found no changes in the total energy within our statistical error of $0.5 \mathrm{~m} E_{\mathrm{h}}$.

\section{Machine-learning with GAP}

The GAP machine-learning techniques that we have developed for representing and correcting 1B and 2B DFT errors of molecular systems ${ }^{57}$ are a particular form of the more general GAP techniques ${ }^{56}$ that have been applied to a variety of other types of system. The GAP corrections employed here are more accurate than the ones that we reported recently for water. ${ }^{57}$

We summarize briefly the GAP procedure. For a system of molecules whose configuration is specified by a point $\mathbf{R}$ in a many-dimensional configuration space, the GAP algorithm is "trained" with the values $f\left(\mathbf{R}_{n}\right)$ of the energy (or corrections to the energy) at a finite set of configurations $\left\{\mathbf{R}_{n}\right\}$. The rules of Bayesian inference ${ }^{89}$ are then used to compute the most likely values of $f(\mathbf{R})$ at configurations $\mathbf{R}$ not in the training set. The predictions are based on the assumption that $f(\mathbf{R})$ is a "smooth" function of $\mathbf{R}$, the correlation between the values of $f(\mathbf{R})$ and $f\left(\mathbf{R}^{\prime}\right)$ at different points being characterized by a Gaussian covariance function $C\left(\mathbf{R}, \mathbf{R}^{\prime}\right)$. The most likely value $f(\mathbf{R})$ is then given by

$$
f(\mathbf{R})=\sum_{n}^{\text {data }} C\left(\mathbf{R}, \mathbf{R}_{n}\right) \alpha_{n},
$$

where the coefficients $\alpha_{n}$ are given by inversion of the linear equations:

$$
f\left(\mathbf{R}_{m}\right)=\sum_{n}^{\text {data }}\left[C\left(\mathbf{R}_{m}, \mathbf{R}_{n}\right)+\epsilon \delta_{m n}\right] \alpha_{n},
$$

where $\delta_{m n}$ is the Kronecker delta, and the diagonal shift $\epsilon$ is included to regularize the linear algebra.

The correction of 1B errors of any chosen DFT approximation is a straightforward matter, since the space of configurations $\mathbf{R}$ is only 3-dimensional. Many methods would suffice to make the correction, but it is convenient to use a GAP-like approach, with the training data being the errors of the $1 \mathrm{~B}$ DFT energy $E_{1 \mathrm{~B}}(\mathrm{DFT})$ on a uniform grid in the space of O$\mathrm{H}$ bond lengths $r_{1}, r_{2}$ and $\mathrm{H}-\mathrm{O}-\mathrm{H}$ bond angle $\theta$. In practice, we take $0.8 \AA<r_{1}, r_{2}<1.15 \AA$ at intervals of $0.025 \AA$ and $72.5^{\circ}<\theta<127.5^{\circ}$ at $5^{\circ}$ intervals, the DFT energy values being computed using the MOLPRO package ${ }^{65,66}$ with AV5Z basis sets. To compute the 1B DFT errors, we use benchmark values of $E_{1 \mathrm{~B}}$ given by the standard Partridge-Schwenke representation, ${ }^{90}$ which is essentially exact for present purposes. 

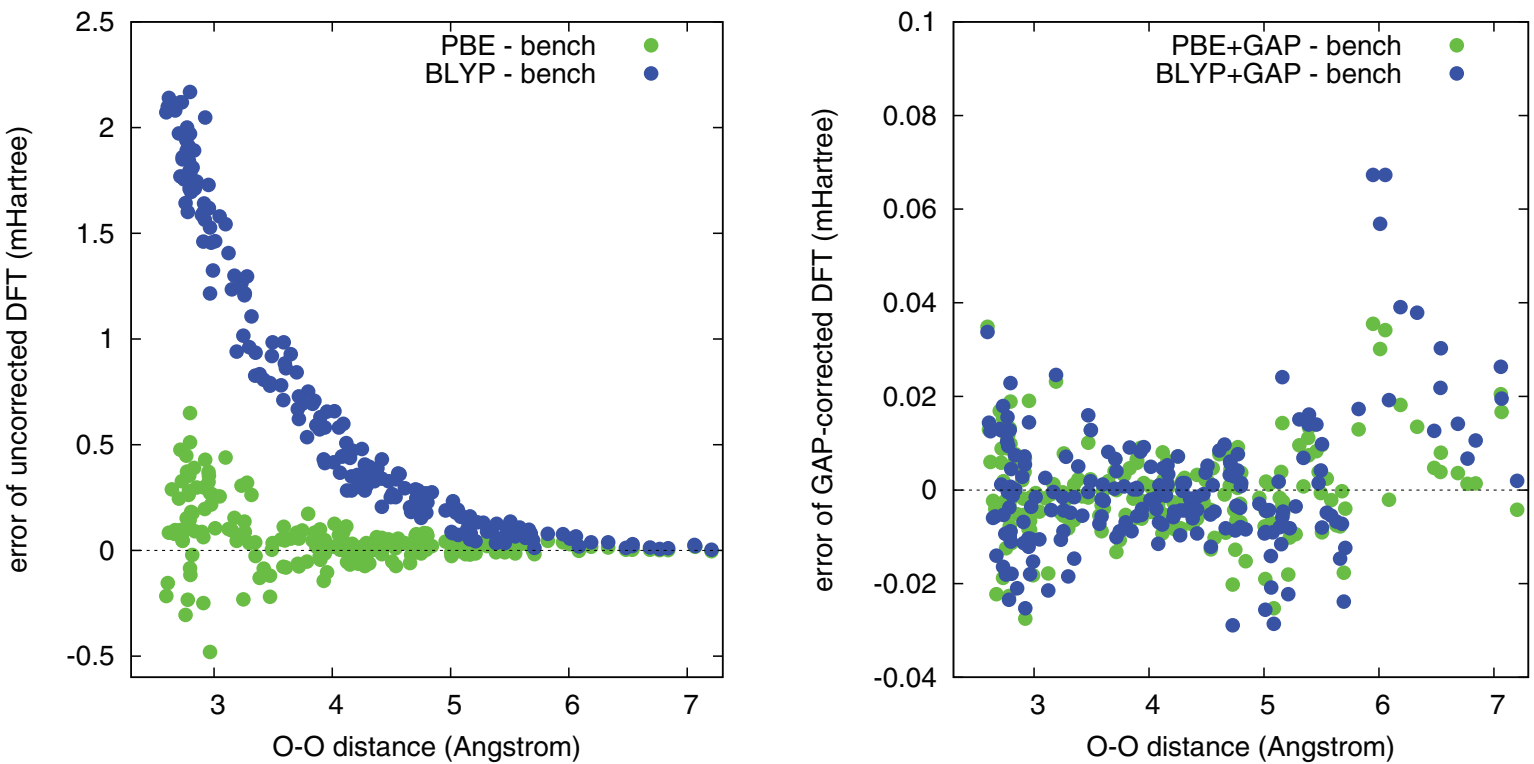

FIG. 1. Left panel: Errors of 2-body energy of $\mathrm{H}_{2} \mathrm{O}$ dimer with BLYP and PBE functionals relative to CCSD(T) benchmarks as function of O-O distance. Calculations are for sample of 198 dimer configurations drawn from a classical m.d. simulation of bulk water at ambient conditions. Right panel: Residual errors of the approximations obtained by adding GAP 2-body corrections to PBE and BLYP. Units: $\mathrm{m} E_{\mathrm{h}}$.

The 2B error of a particular DFT approximation is a function in the space of configurations of a water dimer. As described elsewhere, ${ }^{57}$ we ensure rotational and translational symmetry by working with the space of 15 interatomic distances $\mathbf{R} \equiv\left\{\left|\mathbf{r}_{i}-\mathbf{r}_{j}\right|\right\}$, where $\mathbf{r}_{i}$ are the atomic positions. To impose invariance with respect to interchange of identical atoms, we symmetrize the covariance function over permutations of these atoms. The GAP representation of the correction energy $\Delta E_{2 \mathrm{~B}} \equiv E_{2 \mathrm{~B}}(\mathrm{CCSD}(\mathrm{T}))-E_{2 \mathrm{~B}}(\mathrm{DFT})$ to be added to the DFT energy is constructed in two stages. In the first stage, we create a GAP representation of the difference $E_{2 \mathrm{~B}}(\mathrm{MP} 2 / \mathrm{AVTZ})-E_{2 \mathrm{~B}}(\mathrm{DFT} / \mathrm{AVQZ})$, where the notation indicates that we use Dunning correlation-consistent basis sets, with the augmented quadruple-zeta set being used for DFT and augmented triple-zeta for second-order Møller-Plesset energies. With these basis sets, the DFT energies are within $\sim 20 \mu E_{\mathrm{h}}$ of the CBS limit, but the MP2 energies are only approximate. In the second stage, we correct for the basis-set errors of MP2 and also for the difference between $\operatorname{CCSD}(\mathrm{T})$ and MP2. The correction for basis-set errors of $E_{2 \mathrm{~B}}(\mathrm{MP} 2 / \mathrm{AVTZ})$ employs values of the difference $E_{2 \mathrm{~B}}(\mathrm{MP} 2-\mathrm{F} 12 / \mathrm{AVQZ})-$ $E_{2 \mathrm{~B}}(\mathrm{MP} 2 / \mathrm{AVTZ})$, where MP2-F12 indicates the use of explicitly correlated methods $;{ }^{6-70}$ we add to this the difference $E_{2 \mathrm{~B}}(\mathrm{CCSD}(\mathrm{T})-\mathrm{F} 12 / \mathrm{AVTZ})-E_{2 \mathrm{~B}}(\mathrm{MP} 2-\mathrm{F} 12 / \mathrm{AVTZ})$. The training set of dimer configurations for the data used in the first stage of GAP correction is a set of 9040 configurations, consisting of sub-sets of 6000, 1000, and 2040 configurations. The first of these subsets consists of dimer configurations drawn at random from the classical molecular dynamics (m.d.) simulation of liquid water at ambient conditions described in Ref. 28 , subject to the condition that the oxygenoxygen distance $R_{\mathrm{OO}}$ is less than $4.5 \AA$. The second sub-set is drawn from the same m.d. simulation, subject to the condition that $R_{\mathrm{OO}}$ is between 4.5 and $6.0 \AA$. The final sub-set of 2040 configurations is drawn from a simulation of a water dimer in a confining potential performed with first-principles m.d. at a temperature of $4000 \mathrm{~K}$; these configurations are included to ensure good sampling of the dimer energy surface at small $R_{\mathrm{OO}}$ values. The configuration set for the second stage of GAP correction is a set of 1507 configurations created simply by taking every sixth configuration from the set of 9040 configurations. The basis sets used here are significantly larger than those used in our recent work, ${ }^{57}$ and the GAP corrections generated here are of correspondingly higher quality.

To illustrate the high accuracy achieved by the GAP corrections, we show in Fig. 1 the errors of the uncorrected and corrected BLYP and PBE approximations for the 2B energy of a thermal sample of 198 configurations of the water dimer. These configurations were drawn from exactly the same classical molecular dynamics simulation of ambient liquid water referred to above, but the configurations are entirely independent of those used in training the GAP algorithm. The errors of the approximations are obtained by computing the difference between their $2 \mathrm{~B}$ energies and the benchmark $2 \mathrm{~B}$ energies given by $\mathrm{CCSD}(\mathrm{T})$ close to the CBS limit (see above). The substantial 2B errors of uncorrected BLYP and PBE seen in the left panel of Fig. 1 have been thoroughly discussed in previous papers. ${ }^{28,42}$ The important point here is that for both functionals the GAP corrections reduce the errors to extremely small values of considerably less than $\sim 0.1 \mathrm{~m} E_{\mathrm{h}}$ over the whole range of O-O separations. This means that if we subtract our GAP representation of the $1 \mathrm{~B}$ and $2 \mathrm{~B}$ errors from the DFT energies of any water system, the remaining errors are entirely B2B errors.

\section{RESULTS}

We start the presentation of our results by illustrating how our many-body analysis works for the prism, cage, book, and ring isomers of the water hexamer (see Fig. 2). This forms 


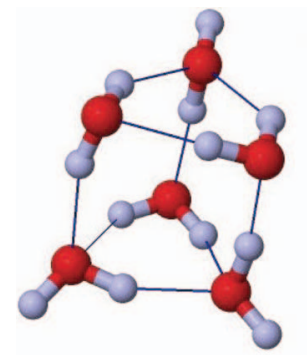

(a)

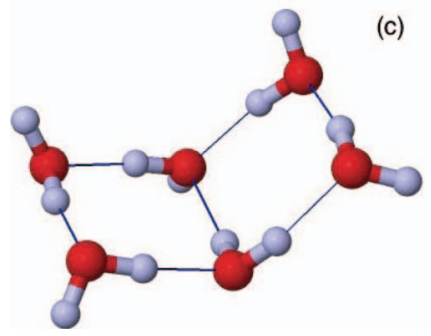

(c)

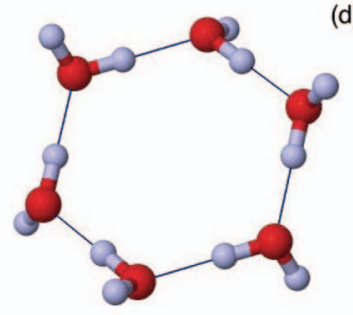

FIG. 2. The four isomers of the $\mathrm{H}_{2} \mathrm{O}$ hexamer for which we analyze DFT errors of the binding energies: (a) prism; (b) cage; (c) book; and (d) ring. Red and gray spheres represent $\mathrm{O}$ and $\mathrm{H}$ atoms, with connecting lines showing hydrogen bonds.

a convenient test case, since the hexamer has been thoroughly studied by many previous authors, ${ }^{29,39,91-93}$ its energetics is very precisely known, ${ }^{94}$ and there have been previous many-body analyses ${ }^{28,29,42}$ of the DFT errors in the binding energies of its isomers. There is an important reason why the water hexamer has received so much attention. For smaller clusters, the most stable isomers have ring-like geometries, but from the hexamer onwards compact geometries are more stable, so that in the hexamer itself there is a rather subtle energy competition between different geometries. Highly converged $\operatorname{CCSD}(\mathrm{T})$ calculations ${ }^{94}$ show that the compact prism and cage isomers are more stable than the extended book and ring, but standard DFT approximations wrongly make the extended geometries more stable. ${ }^{28,29}$ We show here how our GAP $1 \mathrm{~B}$ and $2 \mathrm{~B}$ corrections allow the B2B errors of DFT approximations to be clearly separated.

We compare in Fig. 3 the total binding energies of the isomers computed with BLYP, PBE, and $\operatorname{CCSD}(T)$. The atomic positions used for all the calculations correspond to the equilibrium configurations calculated by Santra et al. ${ }^{29}$ using the MP2 approximation with aug-cc-pVTZ basis sets. The BLYP and PBE energies are those reported in our recent work, ${ }^{28}$ while the $\operatorname{CCSD}(\mathrm{T})$ energies were computed as described above. As expected from previous work, ${ }^{28,29,42}$ BLYP is seriously under-binding and its errors become rapidly more positive as we pass from extended (ring, book) to compact (cage, prism) isomers. Quantitatively, BLYP makes the prism and ring isomers under-bound by 13.3 and $6.8 \mathrm{~m} E_{\mathrm{h}}$ respectively. On the other hand, PBE is somewhat over-binding, but again its errors become more positive (less negative) as we go to more compact geometries. With PBE, the prism and ring are over-bound by 0.5 and $4.6 \mathrm{~m} E_{\mathrm{h}}$.

If we now eliminate the $1 \mathrm{~B}$ and $2 \mathrm{~B}$ errors by using our GAP representations to subtract them from the total energies, then the errors of the resulting approximations (we denote the new approximations by BLYP-2 and PBE-2) are by
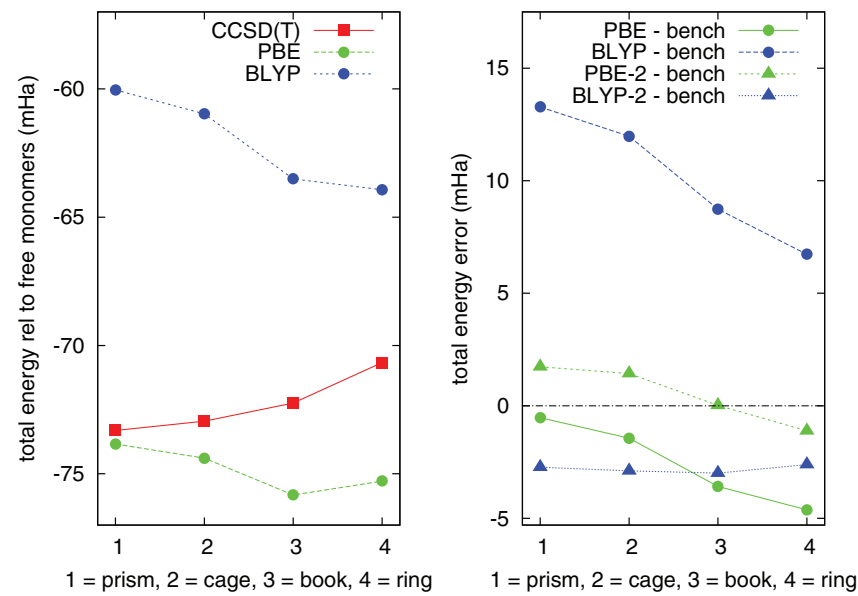

FIG. 3. Left panel: Total energies of isomers of the $\mathrm{H}_{2} \mathrm{O}$ hexamer relative to free monomers from PBE, BLYP, and benchmark CCSD(T) calculations, Right panel: Errors of total energy of PBE and BLYP and 1- and 2-body corrected PBE-2 and BLYP-2. Units: $\mathrm{m} E_{\mathrm{h}}$

definition B2B errors. As shown in Fig. 3, the errors of BLYP2 are negative but almost constant, so that BLYP-2 is somewhat overbound, but it predicts excellent relative energies of the isomers, as reported in recent work. ${ }^{28,57}$ Quantitatively, we find that its errors relative to the $\operatorname{CCSD}(\mathrm{T})$ benchmarks are $-2.7,-2.8,-3.0$, and $-2.6 \mathrm{~m} E_{\mathrm{h}}$ for the prism, cage, book, and ring isomers. The errors of PBE-2 are smaller than those of uncorrected PBE, but they are far from negligible, so that the relative energies with PBE-2 are still poor. The errors of PBE-2 are $1.7,1.5,0.0$, and $-1.1 \mathrm{~m} E_{\mathrm{h}}$ for the prism, cage, book, and ring. This shows that the erroneous stability ordering with BLYP is mainly due to the excessive $2 \mathrm{~B}$ repulsion of BLYP seen in Fig. 1, while with PBE both $2 \mathrm{~B}$ and B2B errors are important, as pointed out before. ${ }^{28,42}$

We now turn to the energetics of ice structures, which give striking evidence of the difficulties facing standard firstprinciples methods. ${ }^{23-26}$ Essentially the same analysis that we used for the hexamers can be used to determine the contribution of many-body errors to the total DFT errors for the cohesive energies of ice structures. Ice has a complicated phase diagram, with no less than 15 known crystal structures, ${ }^{95}$ but we study here only the proton-ordered structures XI, II, XV, and VIII forming the sequence of increasingly compact structures found at low temperatures as pressure increases from 0 to $\sim 20 \mathrm{kbar}$. The errors of DFT approximations for these and other structures have recently been studied in detail, ${ }^{23,26}$ and it was shown that the energies predicted by standard DFT methods increase much too fast from extended to compact structures. We illustrate the problem with the energies in Fig. 4, where we show our own calculated cohesive energies with BLYP and PBE compared with experimental values. ${ }^{96}$ Zero-point energies have been removed from the experimental values, as described in Ref. 23, and the resulting "corrected" experimental cohesive energies are almost identical to those given by quantum Monte Carlo calculations. ${ }^{23}$

Our GAP representations of the $1 \mathrm{~B}$ and $2 \mathrm{~B}$ errors of BLYP and PBE are readily computed in periodic boundary conditions, as explained in Ref. 57. We show in Fig. 4 the errors of the uncorrected and corrected approximations for 

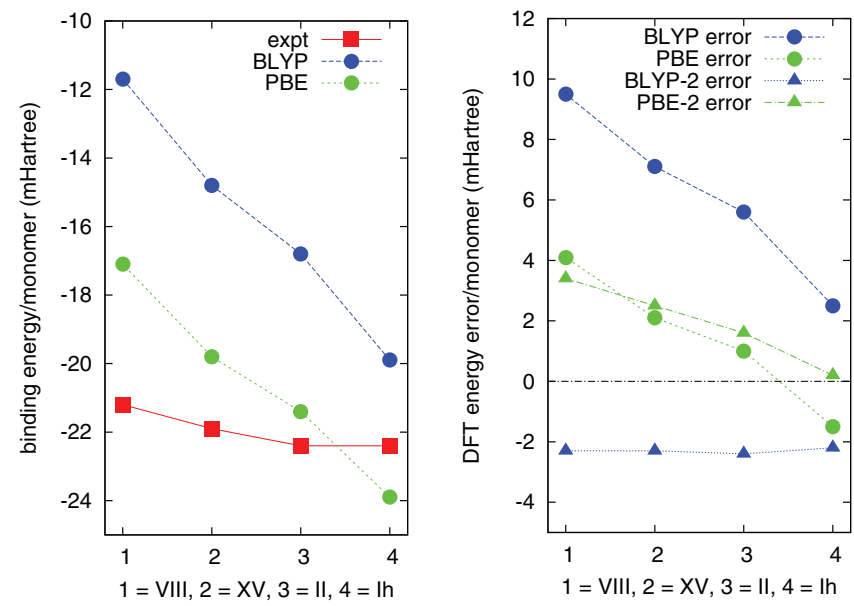

FIG. 4. Left panel: Energies of ice structures relative to free monomers from PBE, BLYP and experiment. Right panel: Errors of PBE and BLYP and 1and 2-body corrected PBE-2 and BLYP-2 energies. Energy units: $\mathrm{m} E_{\mathrm{h}}$.

the cohesive energies of the XI, II, XV, and VIII structures. The picture resembles what we saw for the hexamers. Uncorrected BLYP becomes increasingly under-binding as we go from extended to compact structures, but its corrected version BLYP-2 has almost constant negative errors, so that its relative energies are very good, as reported elsewhere. ${ }^{57} \mathrm{By}$ contrast, the corrected version PBE-2, while better than PBE itself, still gives substantial errors. This implies that for BLYP the problem with relative energies stems mainly from its systematically under-binding $2 \mathrm{~B}$ interaction, but for PBE both $2 \mathrm{~B}$ and $\mathrm{B} 2 \mathrm{~B}$ errors are important.

The energy changes with increasing compactness along the series XI, II, XV, and VIII can be understood in more detail. In all these structures, each $\mathrm{H}_{2} \mathrm{O}$ monomer is hydrogenbonded to four first neighbors at O-O distances of $\sim 2.7 \AA .{ }^{95}$ In XI (the proton-ordered form of the Ih structure of common ice), the monomers form a tetrahedral network, the secondneighbors being at the large distance of $4.5 \AA$. From XI to II, XV, and VIII, the hydrogen-bonded first-neighbor distances change only slightly, but the second-neighbor distances contract dramatically, until in VIII each monomer has eight neighbors at almost equal distances of $\sim 2.8 \AA$, four of which are unbonded to the central monomer. ${ }^{95}$ The close approach of monomers that are not $\mathrm{H}$-bonded to each other in the compact structures appears to be implicated in the large DFT errors, as has been pointed out before (e.g., Ref. 15).

To further probe DFT errors in describing the close approach of non-H-bonded monomers, we have studied two clusters cut from the ice VIII crystal. The first is a nonamer consisting of an $\mathrm{H}_{2} \mathrm{O}$ molecule and its eight neighbors (Fig. 5), and we examine the energy variation when the $\mathrm{H}-$ bonded neighbors are held fixed but the four unbonded neighbors are moved radially outward. The second cluster is a decamer composed of two pentamers, each pentamer consisting of an $\mathrm{H}_{2} \mathrm{O}$ molecule and its four $\mathrm{H}$-bonded neighbors, the two central monomers in the pentamers being each other's non- $\mathrm{H}-$ bonded neighbors in ice VIII (Fig. 5). For this second cluster, we examine the energy variation when the pentamers are rigidly displaced relative to each other along the line joining
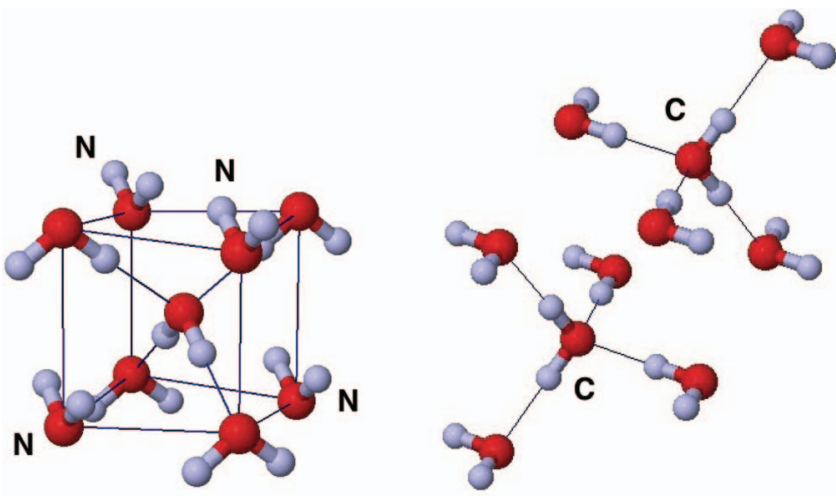

FIG. 5. Nonamer (left panel) and decamer (right panel) cut from ice VIII structure. For each cluster, four configurations are created. For the nonamer, this is done by displacing the non-H-bonded monomers marked " $\mathrm{N}$ " radially outward by $0.0,0.5,1.0$, and $1.5 \AA$. For the decamer, the configurations are created by displacing the pentamer fragments having central monomers " $\mathrm{C}$ " along the axis joining them so that their separation is increased by $0.0,0.5$, 1.0 , and $1.5 \AA$.

the non-H-bonded central monomers. The idea in both cases is to keep H-bonds unchanged but to vary the distances between non-H-bonded monomers. For each cluster, we consider four configurations. The first (configuration 1) has exactly the geometry extracted from ice VIII, and the other three configurations are generated by displacing the moving fragments in successive steps of $0.5 \AA$, so that the distance between non-H-bonded $\mathrm{O}$ atoms increases from $c a .2 .7 \AA$ in configuration 1 to $c a .4 .2 \AA$ in configuration 4 .

Figure 6 reports the DMC benchmark and DFT energies of the two clusters. The benchmark binding energies (left panels) show that there is a significant lowering of energy as the separated fragments approach each other, particularly for the decamer, which shows a stabilization of over $10 \mathrm{~m} E_{\mathrm{h}}$ $(\simeq 300 \mathrm{meV})$. However, the stabilization is not correctly reproduced by either PBE or BLYP. For the nonamer, as we pass from configuration 4 to configuration 1, there should be a stabilization of $\sim 2 \mathrm{~m} E_{\mathrm{h}}$, instead of which there is a destabilization of $\sim 8 \mathrm{~m} E_{\mathrm{h}}$ with PBE and $\sim 17 \mathrm{~m} E_{\mathrm{h}}$ with BLYP. In the case of the decamer, there should be a large stabilization of $\sim 12 \mathrm{~m} E_{\mathrm{h}}$, but there is no stabilization with PBE and a destabilization of $\sim 5 \mathrm{~m} E_{\mathrm{h}}$ with BLYP. The qualitative similarity with the incorrect DFT trends for the ice structures implies that essentially the same DFT errors that give such poor predictions for ice can be observed in relatively small clusters.

Comparing the errors of PBE and BLYP before and after GAP correction for 2B errors (right panels of Fig. 6), we see again the pattern that we found for the hexamers and the ice structures. The BLYP-2 approximation is somewhat overbinding, with an error that is fairly constant, particularly for the nonamer. In the case of PBE, correction for $2 \mathrm{~B}$ errors brings a significant improvement, but does not eliminate the problem, so that a substantial part of the error is due to B2B effects.

\section{DISCUSSION AND CONCLUSIONS}

Our results show that DFT difficulties in treating water systems are partly due to the wrong description of 

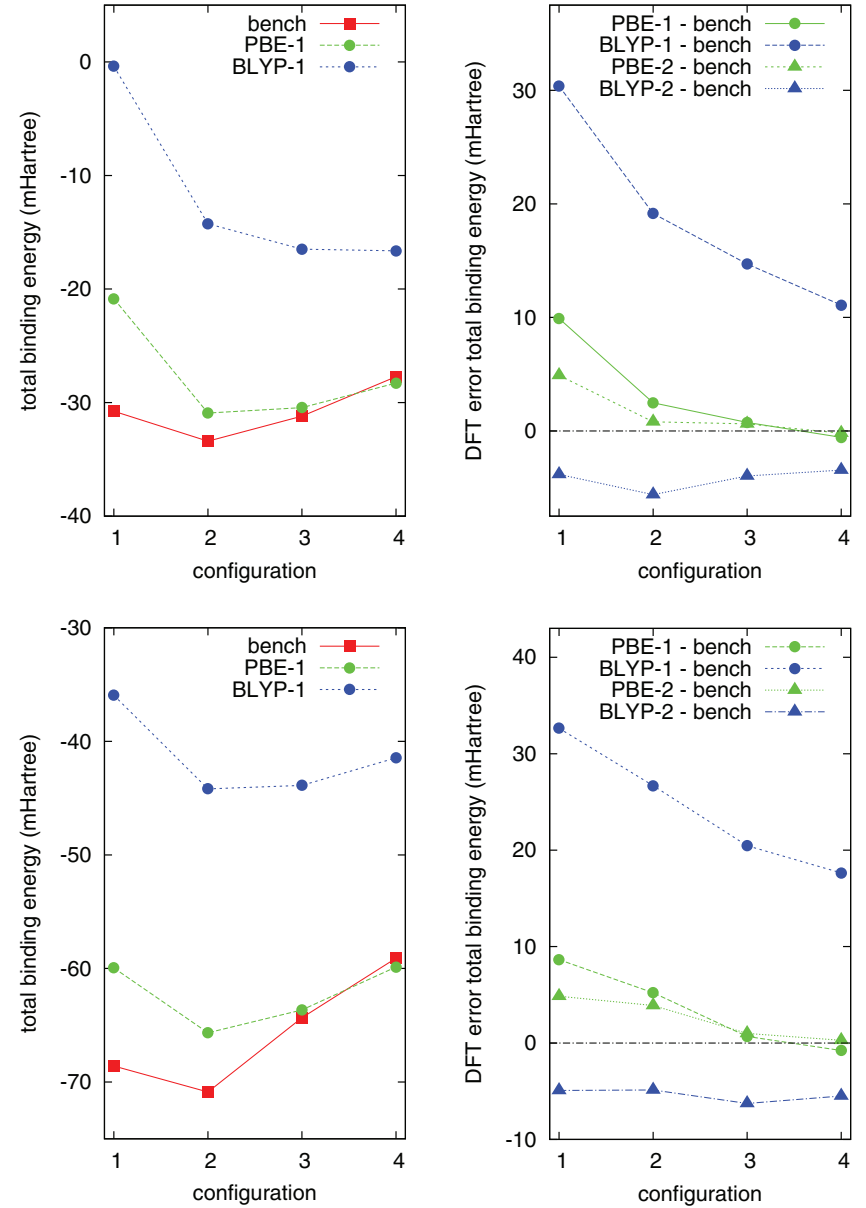

FIG. 6. Left panels: Total binding energies of the four nonamer (upper) and decamer (lower) configurations derived from the ice VIII structure computed with DMC, PBE-1, and BLYP-1; fragments move outward from configuration 1 to configuration 4. Right panels: Errors of the total energy relative to DMC wtih PBE-1, BLYP-1, PBE-2, and BLYP-2 for the nonamer (upper) and decamer (lower) configurations. Energy units: $\mathrm{m} E_{\mathrm{h}}$.

many-body energies. This is not an entirely new result, since it has been emphasized before that the incorrect stability ordering of isomers of the hexamer predicted by some standard DFT functionals-PBE is an example - is partly due to beyond-2-body (B2B) errors. ${ }^{28,42}$ However, the present work shows that the same effects occur even more strongly for ice structures, since correction of both BLYP and PBE for 1- and 2-body errors still leaves substantial errors in their energies. This is important, because there has been a considerable recent effort to correct DFT approximations for water and ice by adding empirical "dispersion-correction" potentials acting between pairs of water molecules. ${ }^{13,14,17,19,24}$ Purely 2-body corrections of this kind can indeed improve the description of liquid water, ${ }^{13,14,17}$ but our results suggest that they cannot be fully successful unless they are added to a DFT approximation that is accurate for the B2B part of the energy. The same comment does not necessarily apply to more sophisticated dispersion-inclusive techniques in which non-local correlation responds to changes of electron density, ${ }^{15,16,18,23,26,33}$ but even so the treatment of B2B energies by these techniques clearly merits further study.
It is noteworthy that the errors of BLYP and PBE for water systems are completely different. The very different character of their $2 \mathrm{~B}$ errors is well known: ${ }^{28,42,59}$ BLYP is systematically too repulsive (insufficiently attractive) for the water dimer over a wide range of $\mathrm{O}-\mathrm{O}$ separations, while $\mathrm{PBE}$ gives a rather accurate binding energy at the global minimum of the dimer. However, we are not aware that the strongly contrasting nature of their B2B errors has been emphasized before. The fact that BLYP becomes significantly overbinding for a range of water systems after correction for its $1 \mathrm{~B}$ and $2 \mathrm{~B}$ errors was shown in recent work, ${ }^{57,100}$ where it was noted that this overbinding is fairly constant if one compares different isomers of the hexamer or different ice structures. (See also Ref. 52, where it was shown that B3LYP, a hybrid version of BLYP, exaggerates the cooperative strengthening of hydrogen bonding in HF clusters.) However, we have seen here that the B2B errors of PBE vary quite strongly across both the hexamers and the ice structures. This appears to indicate that the physical mechanisms underlying the $\mathrm{B} 2 \mathrm{~B}$ errors can be very different for different DFT approximations.

We noted in the Introduction that in GGA approximations such as BLYP and PBE some of the 2-body errors in the interactions between closed-shell molecules are closely related to the behavior of the exchange-enhancement factor at large reduced gradients. There is a connection here with self-interaction errors and the fact that conventional DFT approximations make the tails of electron distributions too diffuse. One promising way of addressing this problem is based on the introduction of atom-centered potentials designed to correct the density distribution, as proposed by Torres and DiLabio $^{97}$ and independently by von Lilienfeld et al. ${ }^{98}$ It has been demonstrated that this approach can greatly improve the prediction of dimer binding energies. ${ }^{99}$ If, as seems possible, some of the many-body errors revealed in the present work have a similar origin, it may be that correction of the density distribution using these methods could help to cure these errors. This is an interesting direction for future study.

It is an important question whether the errors of DFT for solid and liquid condensed-phase water are related to those exhibited by small clusters. Our results suggest that there is a close relationship. In both the isomers of the hexamer and the ice structures, uncorrected BLYP is seriously underbinding, and the degree of underbinding increases strongly as we pass from extended to compact structures. In both systems, the underbinding arises from the excessive $2 \mathrm{~B}$ repulsion of BLYP shown by the dimer. The B2B error that remains when BLYP is corrected for $1 \mathrm{~B}$ and $2 \mathrm{~B}$ errors leads to overbinding in both the hexamers and the ice structures, and this overbinding is almost constant across both kinds of system, so that their relative energies are well predicted. These facts were already noted in our recent work on GAP methods for water. ${ }^{57}$ In a separate study, ${ }^{100}$ we have shown that essentially the same errors of uncorrected and corrected BLYP appear in thermal-equilibrium nano-droplets and even in liquid water. The present work reveals that the errors of uncorrected and corrected PBE also take very similar forms for the hexamers and the ice structures, even though these forms differ strongly from what is found with BLYP. The errors of uncorrected PBE destabilize the compact hexamers and ice structures too much 
compared with the extended structures. Correction for 1B and $2 \mathrm{~B}$ errors reduces the incorrect destabilization, but does not cure the problem, and the remaining B2B errors have essentially the same form for both hexamers and ice. We have also seen here that very similar patterns of errors emerge for the nonamer and decamer clusters cut from ice VIII.

The present work is not primarily about liquid water, but we offer some tentative suggestions about its possible relevance to DFT errors for the liquid, and particularly the underestimation of equilibrium density and the over-structuring produced by both BLYP and PBE. It has been noted before ${ }^{15}$ that these errors may appear because the DFT approximations unduly disfavor the close approach of non-hydrogen-bonded pairs of monomers, an effect that would also explain their erroneous predictions for the relative energies of ice structures. In the case of BLYP, we have seen that its excessive 2B repulsion does make the close approach of non-hydrogen-bonded monomers energetically unfavorable in ice, and correction for this yields good relative energies. This suggests that for BLYP the reported attempts to improve the liquid properties by adding 2B attractive potentials ${ }^{14,17,19}$ are soundly based, though the amount of $2 \mathrm{~B}$ correction may not always have been optimal. With PBE, by contrast, our analysis indicates that $\mathrm{B} 2 \mathrm{~B}$ errors are a major cause of the problem, so that the addition of 2B attractive potentials would not by itself provide an adequate solution. Such 2B "corrections" would in any case tend to worsen the description of the water dimer.

The nature of the many-body errors of DFT approximations for water and other molecular systems requires further analysis. Errors of monomer polarizability may be significant, but it seems likely that other sources of many-body error also contribute. For example, the large enhancement of dipole moment and the associated redistribution of electron density when the water monomer passes from the gas phase to condensed phases might well lead to errors of exchange-repulsion or non-local correlation that differ from those shown by the water dimer in free space. (The importance of many-body contributions other than those accounted for by polarizability has also been recognized in the development of parameterized interaction models. ${ }^{101}$ ) Recently developed forms of embedded many-body expansion ${ }^{102,103}$ may perhaps provide a framework for analyzing such effects.

In summary, we have presented a many-body analysis of DFT errors for the energetics of water clusters and ice structures. The analysis employs GAP machine-learning techniques to correct almost exactly for 1- and 2-body errors, so that beyond-2-body errors can be cleanly separated. Taking the BLYP and PBE approximations as examples, we find that beyond-2-body errors are substantial in both cases but have very different characteristics. The results imply that a fully satisfactory description of water systems can only be provided by techniques that give an accurate account of the many-body components of the energy.

\section{ACKNOWLEDGMENTS}

A.P.B. was supported by a Junior Research Fellowship at Magdalene College, Cambridge. G.C. was supported by the Office of Naval Research under grant N000141010826, and by EU-FP7-NPM grant 229205 ADGLASS. We used resources of the Oak Ridge Leadership Computing Facility in the National Center for Computational Sciences at ORNL, which is supported by the Office of Science of the DOE under Contract No. DE-AC05-00OR22725. Access to HECToR, the UK's national high-performance computing service is acknowledged. We thank A. Michaelides for comments on an early version of the manuscript, and we acknowledge helpful comments from one of the referees.

${ }^{1}$ K. Laasonen, M. Sprik, M. Parrinello, and R. Car, J. Chem. Phys. 99, 9080 (1993); C. Lee, D. Vanderbilt, K. Laasonen, R. Car, and M. Parrinello, Phys. Rev. B 47, 4863 (1993); M. E. Tuckerman, K. Laasonen, M. Sprik, and M. Parrinello, J. Phys. Condens. Matter 6, A93 (1994); M. Sprik, J. Hutter, and M. Parrinello, J. Chem. Phys. 105, 1142 (1996).

${ }^{2}$ K. Laasonen and M. L. Klein, Mol. Phys. 88, 135 (1996); E. J. Meijer and M. Sprik, J. Am. Chem. Soc. 120, 6345 (1998); J. Blumberger, L. Bernasconi, I. Tavernelli, R. Vuilleumier, and M. Sprik, ibid. 126, 3928 (2004).

${ }^{3}$ P. J. D. Lindan, N. M. Harrison, and M. J. Gillan, Phys. Rev. Lett. 80, 762 (1998); L. Liu, M. Krack, and A. Michaelides, J. Am. Chem. Soc. 130, 8572 (2008); J. Carrasco, A. Hodgson, and A. M. Michaelides, Nature Mater. 11, 667 (2012)

${ }^{4}$ J. C. Grossman, E. Schwegler, E. W. Draeger, F. Gygi, and G. Galli, J. Chem. Phys. 120, 300 (2004)

${ }^{5}$ M. Allesch, E. Schwegler, F. Gygi, and G. Galli, J. Chem. Phys. 120, 5192 (2004).

${ }^{6}$ E. Schwegler, J. C. Grossman, F. Gygi, and G. Galli, J. Chem. Phys. 121, 5400 (2004).

${ }^{7}$ P. H.-L. Sit and N. Marzari, J. Chem. Phys. 122, 204510 (2005).

${ }^{8}$ H.-S. Lee and M. E. Tuckerman, J. Chem. Phys. 125, 154507 (2006); 126, 164501 (2007).

${ }^{9}$ M. Guidon, F. Schiffmann, J. Hutter, and J. VandeVondele, J. Chem. Phys. 128, 214104 (2008).

${ }^{10}$ T. D. Kühne, M. Krack, and M. Parrinello, J. Chem. Theory Comput. 5, 235 (2009).

${ }^{11}$ S. Yoo, X. C. Zeng, and S. S. Xantheas, J. Chem. Phys. 130, 221102 (2009).

${ }^{12}$ M. J. McGrath, I.-F. W. Kuo, and J. I. Siepmann, Phys. Chem. Chem. Phys. 13, 19943 (2011).

${ }^{13}$ I.-C. Lin, A. P. Seitsonen, M. D. Coutinho-Neto, I. Tavernelli, and U. Röthlisberger, J. Phys. Chem. B 113, 1127 (2009).

${ }^{14}$ J. Schmidt, J. VandeVondele, I.-F. W. Kuo, D. Sebastiani, J. I. Siepmann, J. Hutter, and C. J. Mundy, J. Phys. Chem. B 113, 11959 (2009).

${ }^{15}$ J. Wang, G. Román-Pérez, J. M. Soler, E. Artacho, and M.-V. FernándezSerra, J. Chem. Phys. 134, 024516 (2011).

${ }^{16}$ A. Møgelhøj, A. Kelkkanen, K. T. Wikfeldt, J. Schiøtz, J. J. Mortensen, L. G. M. Pettersson, B. I. Lundqvist, K. W. Jacobsen, A. Nilsson, and J. K. Nørskov, J. Phys. Chem. B 115, 14149 (2011).

${ }^{17}$ R. Jonchiere, A. P. Seitsonen, G. Ferlat, A. M. Saitta, and R. Vuilleumier, J. Chem. Phys. 135, 154503 (2011).

${ }^{18}$ C. Zhang, J. Wu, G. Galli, and F. Gygi, J. Chem. Theory Comput. 7, 3054 (2011).

${ }^{19}$ Z. Ma, Y. Zhang, and M. E. Tuckerman, J. Chem. Phys. 137, 044506 (2012).

${ }^{20}$ D. R. Hamann, Phys. Rev. B 55, R10157 (1997).

${ }^{21}$ P. Feibelman, Phys. Chem. Chem. Phys. 10, 4688 (2008).

${ }^{22}$ I. Hamada, J. Chem. Phys. 133, 214503 (2010).

${ }^{23}$ B. Santra, J. Klimeš, D. Alfè, A. Tkatchenko, B. Slater, A. Michaelides, R. Car, and M. Scheffler, Phys. Rev. Lett. 107, 185701 (2011).

${ }^{24}$ O. Kambara, K. Takahashi, M. Hayashi, and J.-L. Kuo, Phys. Chem. Chem. Phys. 14, 11484 (2012)

${ }^{25}$ Y. Fang, B. Xiao, J. Tao, J. Sun, and J. P. Perdew, Phys. Rev. B 87, 214101 (2013).

${ }^{26}$ B. Santra, J. Klimeš, A. Tkatchenko, D. Alfè, B. Slater, A. Michaelides, R. Car, and M. Scheffler, J. Chem. Phys. 139, 154702 (2013).

${ }^{27}$ J. A. Anderson and G. S. Tschumper, J. Phys. Chem. A 110, 7268 (2006).

${ }^{28}$ M. J. Gillan, F. R. Manby, M. D. Towler, and D. Alfè, J. Chem. Phys. 136, 244105 (2012).

${ }^{29}$ B. Santra, A. Michaelides, M. Fuchs, A. Tkatchenko, C. Filippi, and M. Scheffler, J. Chem. Phys. 129, 194111 (2008). 
${ }^{30}$ A. K. Kelkkanen, B. I. Lundqvist, and J. K. Norskov, J. Chem. Phys. 131, 046102 (2009).

${ }^{31}$ B. Kolb and R. Thonhauser, Phys. Rev. B 84, 045116 (2011).

${ }^{32}$ E. D. Murray and G. Galli, Phys. Rev. Lett. 108, 105502 (2012).

${ }^{33}$ F. Corsetti, E. Artacho, J. M. Soler, S. S. Alexandre, and M.-V. FernándezSerra, "Room temperature compressibility and diffusivity of liquid water from first principles," preprint arXiv:1307.1645 (2013).

${ }^{34}$ J. Klimeš and A. Michaelides, J. Chem. Phys. 137, 120901 (2012).

${ }^{35}$ J. Perdew, K. Burke, and M. Ernzerhof, Phys. Rev. Lett. 77, 3865 (1996).

${ }^{36}$ A. D. Becke, Phys. Rev. A 38, 3098 (1988); C. Lee, W. Yang, and R. Parr, Phys. Rev. B 37, 785 (1988).

${ }^{37}$ B. Santra, A. Michaelides, and M. Scheffler, J. Chem. Phys. 131, 124509 (2009).

${ }^{38}$ J. Ireta, J. Neugebauer, and M. Scheffler, J. Phys. Chem. A 108, 5692 (2004).

${ }^{39}$ E. E. Dahlke, R. M. Olson, H. R. Leverentz, and D. G. Truhlar, J. Phys. Chem. A 112, 3976 (2008).

${ }^{40}$ M. J. McGrath, J. I. Siepmann, I.-F. W. Kuo, C. J. Mundy, J. VandeVondele, J. Hutter, F. Mohamed, and M. Krack, ChemPhysChem 6, 1894 (2005).

${ }^{41}$ S. S. Xantheas, J. Chem. Phys. 100, 7523 (1994); J. M. Pedulla, F. Vila, and K. D. Jordan, ibid. 105, 11091 (1996).

${ }^{42}$ F.-F. Wang, G. Jenness, W. A. Al-Saidi, and K. D. Jordan, J. Chem. Phys. 132, 134303 (2010).

${ }^{43}$ A. J. Stone, The Theory of Intermolecular Forces, 2nd ed. (Oxford University Press, Oxford, 2013).

${ }^{44}$ C. A. Coulson and D. Eisenberg, Proc. R. Soc. London, Ser. A 291, 445 (1966).

${ }^{45}$ E. R. Batista, S. S. Xantheas, and H. Jónsson, J. Chem. Phys. 109, 4546 (1998).

${ }^{46}$ P. L. Silvestrelli and M. Parrinello, Phys. Rev. Lett. 82, 3308 (1999).

${ }^{47}$ Y. S. Badyal, M.-L. Saboungi, D. L. Price, S. D. Shastri, D. R. Haeffner, and A. K. Soper, J. Chem. Phys. 112, 9206 (2000).

${ }^{48}$ M. Sharma, R. Resta, and R. Car, Phys. Rev. Lett. 98, 247401 (2007).

${ }^{49}$ D. Hankins, J. W. Moskowitz, and F. H. Stillinger, J. Chem. Phys. 53, 4544 (1970).

${ }^{50}$ L. González, O. Mó, M. Yáñez, and J. Elguero, J. Mol. Struct.: THEOCHEM 371, 1 (1996).

${ }^{51}$ A. Karpfen, Adv. Chem. Phys. 123, 469 (2002).

${ }^{52}$ L. Rincón, R. Almeida, and D. G. Aldea, Int. J. Quantum Chem. 102, 443 (2005).

${ }^{53}$ M. P. Hodges, A. J. Stone, and S. S. Xantheas, J. Phys. Chem. A 101, 9163 (1997).

${ }^{54}$ L. X. Dang and T.-M. Chang, J. Chem. Phys. 106, 8149 (1997).

${ }^{55}$ P. Ren and J. W. Ponder, J. Phys. Chem. B 107, 5933 (2003).

${ }^{56}$ A. P. Bartók, M. C. Payne, R. Kondor, and G. Csányi, Phys. Rev. Lett. 104, 136403 (2010).

${ }^{57}$ A. P. Bartók, M. J. Gillan, F. R. Manby, and G. Csanyi, Phys. Rev. B 88, 054104 (2013).

${ }^{58}$ T. Helgaker, P. Jorgensen, and J. Olsen, Molecular Electronic-Structure Theory (Wiley, New York, 2000).

${ }^{59}$ B. Santra, A. Michaelides, and M. Scheffler, J. Chem. Phys. 127, 184104 (2007).

${ }^{60}$ D. J. Lacks and R. G. Gordon, Phys. Rev. A 47, 4681 (1993).

${ }^{61}$ Y. Zhang, W. Pan, and W. Yang, J. Chem. Phys. 107, 7921 (1997).

${ }^{62}$ F. O. Kannemann and A. D. Becke, J. Chem. Theory Comput. 5, 719 (2009).

${ }^{63}$ W. Klopper, J. G. C. M. van Duijneveldt-van de Rijdt, and F. B. van Duijneveldt, Phys. Chem. Chem. Phys. 22, 2227 (2000).

${ }^{64}$ G. S. Tschumper, M. L. Leininger, B. C. Hoffman, E. F. Valeev, H. F. Schaeffer III, and M. Quack, J. Chem. Phys. 116, 690 (2002).

${ }^{65}$ H.-J. Werner, P. J. Knowles, G. Knizia, F. R. Manby, M. Schütz et al., MOLPRO, version 2012.1, a package of ab initio programs, 2012, see http://www.molpro.net.

${ }^{66}$ H.-J. Werner, P. J. Knowles, G. Knizia, F. R. Manby, and M. Schütz, Comput. Mol. Sci. 2, 242 (2012).

${ }^{67}$ A. G. Császár, W. D. Allen, and H. F. Schaeffer, J. Chem. Phys. 108, 9751 (1998).

${ }^{68}$ W. Klopper, F. R. Manby, S. Ten-no, and E. F. Valeev, Int. Rev. Phys. Chem. 25, 427 (2006).
${ }^{69}$ H.-J. Werner, T. B. Adler, and F. R. Manby, J. Chem. Phys. 126, 164102 (2007).

${ }^{70}$ T. B. Adler, G. Knizia, and H.-J. Werner, J. Chem. Phys. 127, 221106 (2007).

${ }^{71}$ In the molpro calculations of MP2 and $\operatorname{CCSD}(\mathrm{T})$ energies using explicitly correlated F12 methods, we used the molpro defaults for the basis sets employed for density fitting and resolution of the identity. Our coupledcluster calculations use the CCSD-F12a energy (rather than CCSD-F12b), which is the choice recommended by the molpro Manual for AVTZ atomic-orbital basis sets. We did not employ empirical scaling of the triples contribution that is sometimes used for $\operatorname{CCSD}(\mathrm{T})$ calculations.

${ }^{72}$ T. H. Dunning, J. Chem. Phys. 90, 1007 (1989).

${ }^{73}$ R. A. Kendall, T. H. Dunning, and R. J. Harrison, J. Chem. Phys. 96, 6796 (1992).

${ }^{74}$ S. F. Boys and F. Bernardi, Mol. Phys. 19, 553 (1970); see also T. Helgaker, P. Jørgensen, and J. Olsen, Molecular Electronic-Structure Theory (Wiley, 2000), Chap. 8.5.

${ }^{75}$ See supplementary material at http://dx.doi.org/10.1063/1.4852182 for further information about convergence to the complete basis-set limit.

${ }^{76}$ S. J. Clark, M. D. Segall, C. J. Pickard, P. J. Hasnip, M. J. Probert, K. Refson, and M. C. Payne, Z. Kristallogr. 220, 567 (2005).

${ }^{77}$ I. G. Gurtubay and R. J. Needs, J. Chem. Phys. 127, 124306 (2007).

${ }^{78}$ M. Korth, A. Lüchow, and S. Grimme, J. Phys. Chem. A 112, 2104 (2008).

${ }^{79}$ J. Ma, D. Alfè, A. Michaelides, and E. Wang, J. Chem. Phys. 130, 154303 (2009).

${ }^{80}$ See e.g. W. M. C. Foulkes, L. Mitaš, R. J. Needs, and G. Rajagopal, Rev. Mod. Phys. 73, 33 (2001).

${ }^{81}$ R. J. Needs, M. D. Towler, N. D. Drummond, and P. López-Ríos, J. Phys. Condens. Matter 22, 023201 (2010).

${ }^{82}$ B. M. Austin, D. Y. Zubarev, and W. A. Lester, Chem. Rev. 112, 263 (2012).

${ }^{83}$ R. J. Needs, M. D. Towler, N. D. Drummond, and P. López-Ríos, CASINO 2.12 User Manual (University of Cambridge, Cambridge, 2013), see www.tcm.phys.cam.ac.uk/ mdt26/casino.html.

${ }^{84}$ D. M. Ceperley and B. J. Alder, Phys. Rev. Lett. 45, 566 (1980); P. J. Reynolds, D. M. Ceperley, B. J. Alder, and W. A. Lester, J. Chem. Phys. 77, 5593 (1982).

${ }^{85}$ D. Alfè and M. J. Gillan, Phys. Rev. B 70, 161101 (2004).

${ }^{86}$ J. R. Trail and R. J. Needs, J. Chem. Phys. 122, 014112 (2005); 122, 174109 (2005).

${ }^{87}$ L. Mitaš, E. L. Shirley, and D. M. Ceperley, J. Chem. Phys. 95, 3467 (1991).

${ }^{88}$ P. Giannozzi et al., J. Phys. Condens. Matter 21, 395502 (2009).

${ }^{89}$ D. J. C. Mackay, Information Theory, Inference, and Learning Algorithms (Cambridge University Press, Cambridge, 2005).

${ }^{90}$ H. Partridge and D. W. Schwenke, J. Chem. Phys. 106, 4618 (1997).

${ }^{91}$ Y. Wang, V. Babin, J. M. Bowman, and F. Paesani, J. Am. Chem. Soc. 134, 11116 (2012).

${ }^{92}$ K. Kim, K. D. Jordan, and T. S. Zwier, J. Am. Chem. Soc. 116, 11568 (1994).

${ }^{93}$ E. S. Kryachko, Chem. Phys. Lett. 314, 353 (1999).

${ }^{94}$ D. M. Bates and G. S. Tschumper, J. Phys. Chem. A 113, 3555 (2009).

${ }^{95}$ V. F. Petrenko and R. W. Whitworth, Physics of Ice (Oxford University Press, 1999); C. G. Salzmann, P. G. Radaelli, E. Mayer, and J. L. Finney, Phys. Rev. Lett. 103, 105701 (2009).

${ }^{96}$ E. Whalley, J. Chem. Phys. 81, 4087 (1984).

${ }^{97}$ E. Torres and G. A. DiLabio, J. Phys. Chem. Lett. 3, 1738 (2012).

${ }^{98}$ O. A. von Lilienfeld, I. Tavernelli, and U. Röthlisberger, Phys. Rev. Lett. 93, 153004 (2004).

${ }^{99}$ G. A. DiLabio, E. R. Johnson, and A. Otero-de-la-Roza, Phys. Chem. Chem. Phys. 15, 12821 (2013).

${ }^{100}$ D. Alfè, A. P. Bartók, G. Csányi, and M. J. Gillan, J. Chem. Phys. 138, 221102 (2013)

${ }^{101}$ V. Babin, G. R. Medders, and F. Paesani, J. Phys. Chem. Lett. 3, 3765 (2012); G. R. Medders, V. Babin, and F. Paesani, J. Chem. Theory Comput. 9, 1103 (2013).

${ }^{102}$ P. J. Bygrave, N. L. Allan, and F. R. Manby, J. Chem. Phys. 137, 164102 (2012).

${ }^{103}$ M. J. Gillan, D. Alfè, P. J. Bygrave, C. R. Taylor, and F. R. Manby, J. Chem. Phys. 139, 114101 (2013). 